\title{
Phase of beta-frequency tACS over primary motor cortex modulates corticospinal excitability
}

Citation for published version (APA):

Schilberg, L., Engelen, T., Ten Oever, S., Schuhmann, T., de Gelder, B., de Graaf, T. A., \& Sack, A. T. (2018). Phase of beta-frequency tACS over primary motor cortex modulates corticospinal excitability. Cortex, 103, 142-152. https://doi.org/10.1016/j.cortex.2018.03.001

Document status and date:

Published: 01/06/2018

DOI:

10.1016/j.cortex.2018.03.001

Document Version:

Publisher's PDF, also known as Version of record

Document license:

Taverne

Please check the document version of this publication:

- A submitted manuscript is the version of the article upon submission and before peer-review. There can be important differences between the submitted version and the official published version of record.

People interested in the research are advised to contact the author for the final version of the publication, or visit the DOI to the publisher's website.

- The final author version and the galley proof are versions of the publication after peer review.

- The final published version features the final layout of the paper including the volume, issue and page numbers.

Link to publication

\footnotetext{
General rights rights.

- You may freely distribute the URL identifying the publication in the public portal. please follow below link for the End User Agreement:

www.umlib.nl/taverne-license

Take down policy

If you believe that this document breaches copyright please contact us at:

repository@maastrichtuniversity.nl

providing details and we will investigate your claim.
}

Copyright and moral rights for the publications made accessible in the public portal are retained by the authors and/or other copyright owners and it is a condition of accessing publications that users recognise and abide by the legal requirements associated with these

- Users may download and print one copy of any publication from the public portal for the purpose of private study or research.

- You may not further distribute the material or use it for any profit-making activity or commercial gain

If the publication is distributed under the terms of Article $25 \mathrm{fa}$ of the Dutch Copyright Act, indicated by the "Taverne" license above, 


\title{
Research Report
}

\section{Phase of beta-frequency tACS over primary motor cortex modulates corticospinal excitability}

\author{
Lukas Schilberg ${ }^{a, b, *, 1}$, Tahnée Engelen ${ }^{a, b, 1}$, Sanne ten Oever ${ }^{a, b}$, \\ Teresa Schuhmann ${ }^{a, b}$, Beatrice de Gelder ${ }^{a, b}$, Tom A. de Graaf ${ }^{a, b, 2}$ and \\ Alexander T. Sack ${ }^{a, b, * *, 2}$ \\ ${ }^{a}$ Department of Cognitive Neuroscience, Faculty of Psychology and Neuroscience, Maastricht University, Maastricht, \\ The Netherlands \\ ${ }^{\mathrm{b}}$ Maastricht Brain Imaging Center, Maastricht, The Netherlands
}

\section{A R T I C L E I N F O}

\section{Article history:}

Received 12 September 2017

Reviewed 03 November 2017

Revised 11 January 2018

Accepted 1 March 2018

Action editor Sven Bestmann

Published online 9 March 2018

\section{Keywords:}

Corticospinal excitability

Motor evoked potentials

Neuronal oscillations

Transcranial alternating current

stimulation

Transcranial magnetic stimulation

\begin{abstract}
A B S T R A C T
The assessment of corticospinal excitability by means of transcranial magnetic stimulation-induced motor evoked potentials is an established diagnostic tool in neurophysiology and a widely used procedure in fundamental brain research. However, concern about low reliability of these measures has grown recently. One possible cause of high variability of MEPs under identical acquisition conditions could be the influence of oscillatory neuronal activity on corticospinal excitability. Based on research showing that transcranial alternating current stimulation can entrain neuronal oscillations we here test whether alpha or beta frequency tACS can influence corticospinal excitability in a phase-dependent manner. We applied tACS at individually calibrated alpha- and beta-band oscillation frequencies, or we applied sham tACS. Simultaneous single TMS pulses time locked to eight equidistant phases of the ongoing tACS signal evoked MEPs. To evaluate offline effects of stimulation frequency, MEP amplitudes were measured before and after tACS. To evaluate whether tACS influences MEP amplitude, we fitted one-cycle sinusoids to the average MEPs elicited at the different phase conditions of each tACS frequency. We found no frequencyspecific offline effects of tACS. However, beta-frequency tACS modulation of MEPs was phase-dependent. Post hoc analyses suggested that this effect was specific to participants with low $(<19 \mathrm{~Hz})$ intrinsic beta frequency. In conclusion, by showing that beta tACS influences MEP amplitude in a phase-dependent manner, our results support a potential role attributed to neuronal oscillations in regulating corticospinal
\end{abstract}

\footnotetext{
* Corresponding author. Faculty of Psychology and Neuroscience, Department of Cognitive Neuroscience, Oxfordlaan 55, Maastricht, 6229 EV, The Netherlands.

** Corresponding author. Faculty of Psychology and Neuroscience, Department of Cognitive Neuroscience, Oxfordlaan 55, Maastricht, 6229 EV, The Netherlands.

E-mail addresses: lukas.schilberg@maastrichtuniversity.nl (L. Schilberg), a.sack@maastrichtuniversity.nl (A.T. Sack).

${ }^{1}$ Authors contributed equally to the manuscript; co-first authorship.

2 Authors contributed equally to the manuscript; co-last authorship. 
excitability. Moreover, our findings may be useful for the development of TMS protocols that improve the reliability of MEPs as a meaningful tool for research applications or for clinical monitoring and diagnosis.

() 2018 Elsevier Ltd. All rights reserved.

\section{Introduction}

Non-invasive stimulation of the human brain is widely applied in research and clinical settings. One commonly used application is the assessment of corticospinal excitability. For that, transcranial magnetic stimulation (TMS) over the motor cortex is combined with electromyography (EMG) from peripheral muscles (e.g., hand muscles) to induce and measure motor evoked potentials (MEPs) (Rothwell et al., 1999). The magnitude of such MEPs reflects corticospinal excitability and their evaluation can be of great benefit in both research and clinical applications (Rossini \& Rossi, 1998).

MEPs are usually evaluated at the beginning of most TMS experiments or before TMS treatment to determine stimulation intensity. In addition, measuring MEPs allows for the investigation of intra- and interhemispheric connections and interactions (Bestmann \& Krakauer, 2014), and can provide information about neuroplasticity mechanisms (Moliadze, Fritzsche, \& Antal, 2014; Pascual-Leone, Tormos, \& Keenan, 1998) or specific intervention efficacies (Di Lazzaro et al., 1999). Moreover, MEP assessments can be useful in diagnosis of neurological diseases (Ni \& Chen, 2015), such as Alzheimer's disease (Di Lazzaro et al., 2004; Ferreri et al., 2002), Parkinson disease (Chen, Kumar, Garg, \& Lang, 2001; Lefaucheur, 2005) or multiple sclerosis (Fuhr, Borggrefe-Chappuis, Schindler, \& Kappos, 2001; Kallmann, Facklemann, Toyka, RIekmann \& Reiners., 2006; Rico et al., 2009).

Although widely implemented in research and clinic, such measures of corticospinal excitability display substantial and largely unexplained within- and between-subject variability (Jung et al., 2010; Kiers, Cros, Chiappa, \& Fang, 1993; Rösler, Roth, \& Magistris, 2008). While being a valuable average measurement tool on the group level, this unexplained variability reduces the reliability of corticospinal excitability assessments with TMS, severely hampering its value as a diagnostic or scientific marker for a given individual participant or patient. Revealing factors that modulate corticospinal excitability measures would add to our fundamental understanding of the motor system and human cortical excitability mechanisms. Furthermore, reducing variability could have direct experimental and clinical implications, as the reliability of TMS-induced MEP measures of corticospinal excitability would be increased, thereby both allowing for greater applicability of this technique as well as increasing the validity of corticospinal excitability assessments.

Neuronal networks within and across brain systems routinely engage in synchronized rhythmic activity to establish connectivity between neuronal ensembles and allow for the selection or maintenance of information (Buzsáki \& Draguhn, 2004). When looking at sources of variability in corticospinal excitability measures, it seems that such neuronal oscillations may cause, or reflect, fluctuations in cortical excitability (Mäki \& Ilmoniemi, 2010). In humans, electroencephalography (EEG) can be used to measure oscillatory brain activity at the scalp. The overall strength of oscillations in a particular frequency-band is reflected by the power (directly related to amplitude) of the EEG signal, while its phase reflects the momentary state (e.g., peak or trough) of the ongoing rhythm. Different brain systems inherently seem to operate in different frequency bands. Beta frequency $(13-30 \mathrm{~Hz})$ is the main frequency of the sensorimotor system (Feurra et al., 2011; Kilavik, Zaepffel, Brovelli, Mackay, \& Riehle, 2013; Niedermeyer, 2005) and alpha frequency is suggested to be involved in cortical inhibition (Jensen \& Mazaheri, 2010; Mathewson et al., 2011; Pfurtscheller, Stancák, \& Neuper, 1996).

In the current study, we measured EMG while simultaneously applying TMS and transcranial alternating current stimulation (tACS) to investigate whether tACS at alpha and beta frequency modulates MEP amplitude. tACS is a periodic electrical stimulation of the human cortex, effectively enabling modulation over oscillations (Antal \& Paulus, 2013). Thereby, specific oscillatory frequencies can be entrained in the targeted brain areas (Herrmann, Rach, Neuling, \& Strüber, 2013). When TMS is applied simultaneously to the same target region it is possible to control the timing of the TMS pulse and lock it to particular phases of the entraining tACS signal (Ten Oever et al., 2016; Raco, Bauer, Tharsan, \& Gharabaghi, 2016). Enhancement of the amplitude of intrinsic neuronal oscillations occurs both online during tACS (Fröhlich \& McCormick, 2010) and offline following tACS (Neuling, Rach, \& Herrmann, 2013; Zaehle, Rach, \& Herrmann, 2010). The effects of tACS entrainment over M1 at both alpha and beta frequencies are specific to brain areas immediately underneath and in close proximity to the stimulation electrodes, including pre-and post-central gyri (Witkowski et al., 2016).

We recently developed and validated a setup that allows administration of TMS pulses (or indeed any (multi-) sensory stimulation) time-locked to tACS frequency and phase with sub-millisecond precision (Ten Oever et al., 2016). This setup enables the investigation of how the ongoing tACS signal influences MEP amplitude. Here, TMS pulses were delivered at eight equidistant phases of a sinusoidal tACS signal applied to the motor cortex (Fig. 1A). We administered tACS at either individual peak alpha frequency $(8-13 \mathrm{~Hz})$, individual peak beta frequency $(16-25 \mathrm{~Hz})$, or tACS was simulated (sham tACS). We performed several analyses that directly evaluated whether data revealed a one-cycle oscillatory pattern of MEP amplitudes across the eight TMS-tACS phase conditions. We hypothesized that a causal role of tACS frequency and phase 
A

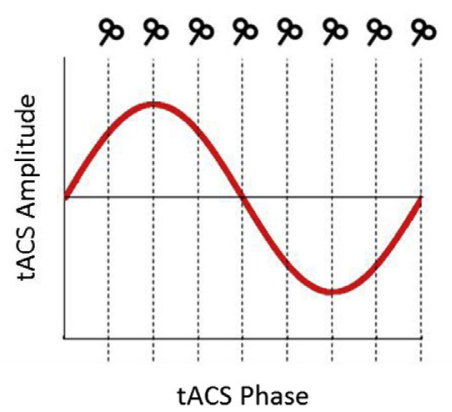

B

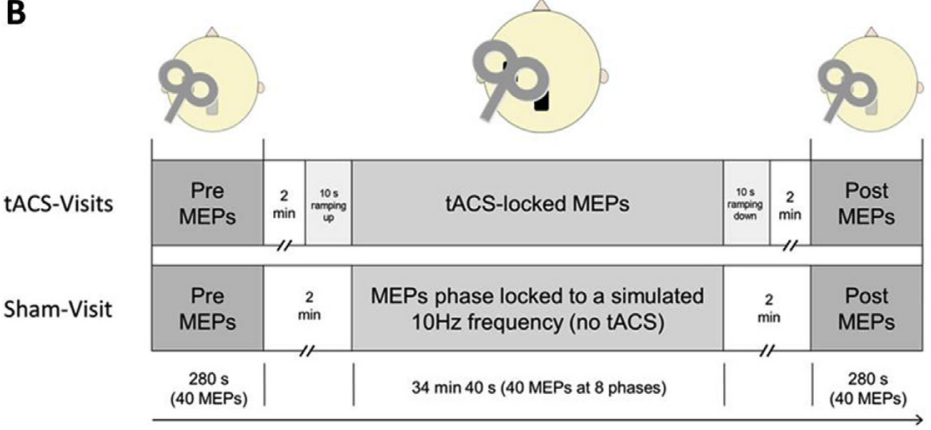

Fig. 1 - A) TMS pulses were administered at eight equidistantly spaced phases of a one-cycle sinusoidal tACS signal applied over M1. The order of TMS administration was randomized with a total of forty pulses per phase. B) Overview of the experimental design. Measures of 40 spTMS-elicited MEPs were conducted 2 min before and after the application of combined tACS-spTMS. During the Alpha-Visit and Beta-Visit this combined protocol consisted of tACS entrainment at either individually predefined alpha or beta frequency together with spTMS phase locked to this tACS frequency. tACS was ramped up for $10 \mathrm{sec}$ before and ramped down for $10 \mathrm{sec}$ after the combined tACS-TMS protocol. During Sham-Visit tACS electrodes were attached, but not connected to the amplifier, while spTMS was applied phase-locked to a simulated $10 \mathrm{~Hz}$ frequency. The order of the visits was counterbalanced across participants.

would lead to mean differences in MEP amplitudes when comparing different frequencies and phase conditions. More stringently, we expected to show tACS frequency-dependence during and after the stimulation by comparing MEP amplitude elicited by TMS-only trials (without tACS) with MEP amplitudes during and after combined tACS-TMS. Finally, we expected tACS signal phase-locking of TMS to results in an oscillatory pattern of MEP amplitudes across the eight TMStACS phase conditions, thereby showing tACS phasedependence of corticospinal excitability.

\section{Methods}

\subsection{Participants}

Fifteen healthy participants completed all three sessions of the experiment $[10$ females, mean age $=24.4( \pm 3.7$ SD) $]$. All participants were right handed and had normal or corrected to normal vision. Participants were screened for TMS safety based on published safety guidelines (Rossi, Hallett, Rossini, \& Pascual-Leone, 2012). All participants gave written informed consent before taking part in the experiment. The study was performed in accordance to the Declaration of Helsinki and it was approved by the local ethical committee.

\subsection{Individual EEG localizers}

To establish optimal individualized stimulation frequencies for tACS, each participant completed a short EEG localizer task at the beginning of the alpha and beta session. EEG data was recorded using BrainAmp MR Plus EEG amplifiers and BrainVision recorder (Brain products, Gilching, Germany) with a sampling rate of $2500 \mathrm{~Hz}$, using a hardware bandpass filter of .1-1000 Hz, a software low pass filter of $250 \mathrm{~Hz}$, and a notch filter at $50 \mathrm{~Hz}$. Three EEG electrodes were placed according to the 10-20 system over C3 (motor cortex), Cz (ground) and right mastoid (reference) using Ten20 paste (Weaver, Colorado). Recording was not started until an impedance of $<20 \mathrm{kOhm}$ was established. During the individual alpha localizer, participants were instructed to sit in a relaxed position with their eyes closed for 5 min while EEG was recorded. For the individual beta localizer, participants were instructed to voluntarily press a button with their right index finger (approximately every $6 \mathrm{sec}$ ) for $10 \mathrm{~min}$ while EEG was recorded. This led to a total of ca. 70 button presses per participant (similar to Romei et al., 2016; Romei, Thut, \& Silvanto, 2016).

\subsection{TMS parameters}

In order to assure stimulation location accuracy throughout each session the BrainVoyager neuronavigation system (Brain Innovation B.V., Maastricht, The Netherlands) was used. Single TMS pulses were applied over M1 in the left hemisphere. Magnetic stimulation was applied before, during, and after tACS stimulation at $120 \%$ of individual resting motor threshold (mean stimulation intensity $=50 \%( \pm 6.1 \mathrm{SD})$ of maximum stimulator output) using a MC-B70 figure-of-eight coil and a Magpro X 100 stimulator (Magventure A/S, Farum, Denmark). Individual motor thresholds were defined as the lowest stimulation intensity needed to evoke an MEP (>.05 mV) in five out of ten pulses. The inter pulse interval was jittered around $6.75( \pm .75)$ sec. The coil was placed tangentially to the scalp with the handle pointing backwards at a $45^{\circ}$ angle to the midline. Placement location was determined based on an optimal position for induction of maximal MEPs in the contralateral target muscle. All delivered pulses were biphasic with the current flowing in an antero-posterior and then postero-anterior (AP - PA) direction in the brain. Each TMS pulse triggered electromyography recordings of the right first dorsal interosseous (FDI) muscle for $100 \mathrm{msec}$ pre-until $150 \mathrm{msec}$ post pulse. To measure EMG of the FDI muscle, 
disposable adhesive silver-chloride surface electrodes (Plaquette $^{\mathrm{TM}}$, Technomed Europe) were placed in a belly-tendon montage with a ground electrode on the wrist. EMG signals were recorded using a Powerlab 4/35 with a Bio Amp system (ADInstruments, Sydney, Australia). The signal was amplified, sampled at $4 \mathrm{k} / \mathrm{s}$, digitized and stored on a computer for offline analysis.

\section{4. tACS parameters}

tACS stimulation was applied using a DC-stimulator plus (NeuroConn, Ilmenau, Germany). After removal of the EEG electrodes, two $3 \times 3 \mathrm{~cm}$ standard tACS electrodes were fixated using conductive EEG gel (Ten20 conductive Neurodiagnostic electrode paste, WEAVER and company, Aurora CO, USA). One tACS-electrode was applied over the predefined individual motor hotspot in the left hemisphere, and the other tACS-electrode was applied on location $\mathrm{Pz}$ based on the international 10-20 system. The intensity of stimulation was fixed for every participant at $1.5 \mathrm{~mA}$ peak-to-peak, with a ramping up and down phase of $10 \mathrm{sec}$. External control of tACS can involve very small DC offsets (here, using the NeuroConn 'Remote' feature) of likely negligible influence. Nevertheless, we here reduced, but not completely removed, these offsets by adapting source files driving both tACS and TMS stimulation (see Ten Oever et al., 2016). During each session the stimulation frequency was set to either individual alpha or beta frequency for each participant. The total time of stimulation was $36.5 \mathrm{~min}$. Positioning of the tACS electrodes was identical in the sham session to ensure similar distances between the TMS coil and the scalp, only the wires were not plugged into the stimulator (not visible for participants). There was no ramping up or down during the Sham-Visit.

\subsection{Procedure}

Participants came in for three sessions (alpha, beta, sham tACS), with the order of conditions being counterbalanced across participants. Alpha and beta tACS sessions started with EEG localizers. EMG and tACS electrodes were placed on the hand and head of the participants. Electrode placement was optimized to yield resting EMG signal below .05 mV (peak-topeak). Neuronavigation emitters were fixated on the participants' heads, which were co-registered to a dummy head in BrainVoyager for accurate and stable coil placement throughout each session. The optimal location for stimulation was determined by systematically searching for the hand hotspot with single TMS pulses over left M1. Once the best location for the stimulation of the FDI muscle was found, one tACS-electrode was placed over this hotspot (see tACS parameters). The TMS coil was fixed into position on top of this electrode using a coil holder, while the participant rested their head in a chinrest. In addition, the coil location was registered in the BrainVoyager Neuronavigation system to ensure the stimulation site did not vary during the session. After the coil was fixed into the optimal position, resting motor threshold was assessed. Before the start of tACS entrainment, a baseline of corticospinal excitability was measured by applying 40 pulses at $120 \%$ of resting motor threshold and recording the corresponding MEPs. Then, the
Table 1 - Overview of the values obtained in the individual alpha and beta EEG localizers per participant that were used for tACS entrainment.

\begin{tabular}{lcc}
\hline Participant & Individual Alpha & Individual Beta \\
\hline 1 & 10.6 & 23.5 \\
2 & 11.4 & 20 \\
3 & 10.2 & 21.25 \\
4 & 10 & 16 \\
5 & 8.6 & 18 \\
6 & 10.6 & 19 \\
7 & 8 & 16 \\
8 & 9.4 & 21 \\
9 & 11.8 & 16.5 \\
10 & 10.8 & 16.75 \\
11 & 10.2 & 25 \\
12 & 10.2 & 16 \\
13 & 10.2 & 16 \\
14 & 11.4 & 19.5 \\
15 & 9.8 & 19.5 \\
\hline
\end{tabular}

tACS stimulation was used to entrain motor cortex oscillations at either individual alpha (mean $=10.2 \mathrm{~Hz}$ ), beta (mean $=18.9 \mathrm{~Hz}$ ) or sham frequency, while single TMS pulses were applied at eight equidistant phases of one oscillatory cycle of the tACS signal (Fig. 1A, see Table 1 for all individual tACS stimulation frequencies). tACS stimulation lasted for a total of 36.5 min while 40 TMS pulses per phase bin were randomly administered. Phase locking of TMS pulses to the tACS signal was established through in house software (Data Streamer), for more details see Ten Oever et al. (2016). After the completion of tACS entrainment, another 40 TMS pulses were applied to assess tACS after-effects on corticospinal excitability. For an overview of the experimental design see Fig. 1B.

\section{Analysis}

\subsection{Individual EEG localizers}

All EEG data were pre-processed and analysed using the FieldTrip toolbox (Oostenveld, Fries, Maris, \& Schoffelen, 2011). The individual beta frequency was determined by measuring the dominant frequency from the cortex $1 \mathrm{sec}$ after voluntary muscle movement (beta rebound) (Jurkiewicz, Gaetz, Bostan, \& Cheyne, 2006; Pfurtscheller et al., 1996; Romei et al, 2016; Romei, Thut, et al, 2016; Witkowski et al., 2016). EEG data of the beta localizer were epoched into -2 to +2 sec time windows around the button press and demeaned. Trials with high variance were removed based on visual inspection. A time-frequency analysis was performed applying wavelets for frequencies ranging from .25 to $30 \mathrm{~Hz}$ in steps of $.25 \mathrm{~Hz}$. The width of the wavelets increased linearly from 2 to 10 cycles. Power values were normalized as a relative change from a baseline period ( -1 to $-.5 \mathrm{sec}$ to button presses). Finally, the peak beta frequency in a frequency range of 14-25 Hz averaging over a .3-.8 sec time window was extracted. Some participants repeated the task as no clear visual peak was present in the initial recording of the voluntary button press task. 
In the individual alpha EEG localizer, participants kept their eyes closed for $5 \mathrm{~min}$. Resulting EEG data were arbitrarily epoched to 5000 msec segments, on which a Fourier transform was done to yield a power spectrum per epoch with a frequency resolution of $0.2 \mathrm{~Hz}$. These spectra were averaged across epochs. Individual alpha frequency was defined as the frequency in the 7-13 $\mathrm{Hz}$ range of the spectrum with the local maximum power value (alpha 'peak').

\subsection{MEP pre-processing}

Neurophysiological data were processed offline. Mean MEP peak-to-peak values were calculated in $\mathrm{mV}$ using Labchart 8 (ADInstruments, Sydney, Australia). To rule out any effects of muscle pre-contraction on MEP amplitude, all trials with prepulse peak-to-peak values deviating more than 3 SDs from the average of all $100 \mathrm{msec}$ pre-pulse values were excluded $(<5 \%$ of trials). Additionally, any trials with MEP peak-to-peak values below $.05 \mathrm{mV}$ were removed. After initial cleaning of the data, outliers deviating more than 3 SDs from the mean MEP values of each condition (10 conditions in total representing each phase bin and pre- and post MEPs) were removed. Average MEPs per phase were normalized for each individual using the average across all phases. To ensure that the EMG signal, and therefore also the MEP results, were not confounded by the concurrent application of tACS, the data was additionally filtered with a high-pass filter at $30 \mathrm{~Hz}$ [falling well above the highest used tACS stimulation frequency of $25 \mathrm{~Hz}$ )] using the FieldTrip Toolbox (one pass, FIR-filter, filter order $=100$ ).

\subsection{The effect of tACS at alpha and beta frequency on corticospinal excitability}

We evaluated the effect of tACS at alpha and beta frequencies on corticospinal excitability by comparing MEPs before, during, and after tACS. We performed a repeated measures ANOVA with time (pre/during/post) and tACS (alpha/beta/ sham) as within subject factors. Individual MEPs, after preprocessing, were averaged per time and tACS condition. Greenhouse-Geisser correction was used in case of violation of the sphericity assumption as indicated by Mauchley's test.

\subsection{The effect of tACS phase on corticospinal excitability}

To assess the effect of tACS phase MEP amplitudes were averaged for each phase bin per participant, and normalized to the individual mean MEP across phase bins. This yielded patterns of MEPs over tACS oscillatory phase bins. If, as hypothesized, tACS phase is relevant for MEP amplitude, then these patterns should depict a single sinusoidal cycle. Thus, the core of our analysis was to fit one-cycle sinusoids on MEP patterns (MEP over the 8 phase bins). We performed different such analyses. In group analyses, we averaged the normalized MEP patterns over participants, per tACS condition, and fit a one-cycle sinusoid on the resulting group pattern. In subject analyses, we fit one-cycle sinusoids on individual MEP patterns, and performed second-level statistical analyses on the measures of fit.

In all cases, one-cycle sinusoids were fit to MEP patterns (per condition alpha/beta/sham) using the Matlab function 'fminsearch' (based on the parameters phase, amplitude and mean). We calculated explained variance (R-squared) measures of best-fitting sinusoids, and multiplied them by the variance of predicted values, to obtain the established 'relevance value' (Fiebelkorn et al., 2011). The relevance value takes into consideration both amplitude and explained variance of sinusoid fits, based on the notion that a sinusoidal pattern with large amplitude and a given goodness of fit is more meaningful than a sinusoidal pattern with the same goodness of fit but small amplitude. We used relevance values for second-level statistical analyses.

Statistical significance of relevance values was primarily tested via permutation tests. In each iteration (1000 iterations), always per participant and per tACS session, the phase condition labels of individual MEP trials were randomly shuffled. Then (randomly ordered) phase bin averages were recalculated and normalized as before. In a first group analysis, individual permuted MEP patterns were averaged over participants to obtain permuted group MEP patterns to which one-cycle sinusoids were fit and relevance values were obtained. The non-permuted (actually obtained) relevance values for alpha/beta/sham conditions were compared to the distributions of permuted relevance values to obtain $p$-values (i.e., proportion of permuted relevance values higher than the non-permuted relevance value). Secondly, to rule out that the observed group-level effects were driven by a minor subset of the subject sample, we performed a cross-validation group analysis for each tACS session. In this analysis, once for each participant, a onecycle sinusoid was fit to the group-averaged MEP pattern excluding that one participant. The phase of the best-fitting sinusoid on this sub-group average result was extracted, and a sinusoid with the same fixed phase (but free amplitude) was fitted to the data of the one excluded participant, yielding a relevance value for that fit. This was repeated for each participant, to obtain an average relevance value for the group. This process was again statistically evaluated in a 1000-iteration permutation test.

These group analyses have the benefit of statistical power, since individual participants' results are averaged into a group result. But they are based on the assumption that, in tACS-TMS, putative sinusoidal patterns of MEPs over phase bins are phase-locked. Randomly phase-shifted individual results could cancel each other out. Additionally, caution should be taken when interpreting the generalizability of the group curve-fitting results, as the curve fitting approach used here corresponds to a fixed effects analysis. For this reason we additionally performed an individual participant analysis. Per tACS session we obtained relevance values from best-fitting sinusoids on individual patterns of MEPs over phase bins. We obtained null distributions (1000 iterations) for each participant/session separately, extracting median permuted relevance values from each. As the data was found to be not normally distributed (according to the Kolmogorov-Smirnov test), the Wilcoxon signed-rank test was performed on the group level, testing observed individual relevance values against associated medians from individual null distributions. Family wise Bonferroni error corrections were applied to each of the separate tests (3 comparisons per test, $\alpha_{\text {bonf }}=.05 / 3$ ). 


\subsection{Post hoc analysis: high versus low beta peaks}

After inspection of the individual beta frequencies obtained in the EEG localizer, we noticed a large distribution of individual peak frequencies within the beta band (between 16 and $25 \mathrm{~Hz}$ ) and performed a median split on individual beta frequencies to divide the sample into two groups with either low- $(<19 \mathrm{~Hz})$ or high-frequency $(>19 \mathrm{~Hz}$ ) beta peaks (see Table 1 for observed individual peak alpha and beta frequencies per participant). Subsequently, we performed post-hoc analyses in which the data was split into two groups, one with a low $[\mathrm{N}=7$, mean frequency $=16.5( \pm .75)]$ and one with a high $[\mathrm{N}=8$, mean frequency $=21.3( \pm 2.2)]$ individual peak beta frequency. All analyses performed on the group level were repeated for these separate groups.

\section{Results}

We first evaluated the causal effect of tACS at individual alpha and beta frequency on corticospinal excitability by comparing average MEPs before, during and after tACS administration (Fig. 2). The repeated measures ANOVA with factors time (pre/ during/post) and condition (alpha/beta/sham) revealed a significant main effect of time $(F(2,28)=7.64, p<.01)$, but no significant main effect of condition $(\mathrm{F}(2,28)=.58, p=.56)$ or time $\times$ condition interaction $(\mathrm{F}(4,56)=1.04, p=.39)$. Bonferroni corrected pairwise comparisons revealed significant differences between both the pre and during $(p<.001)$, and a nearly significant difference between the pre and post measurement $(p=.05)$, showing a general increase of MEP amplitude over time. Results indicate that no frequencyspecific increases in MEP amplitude resulted from tACS entrainment (either alpha or beta).

We then looked at the causal role of tACS phase on corticospinal excitability, by evaluating relevance values for onecycle sinusoid fits to MEPs elicited by TMS at eight equidistant phase bins of ongoing tACS. Group analysis involved sinusoid-fitting to a group average of normalized MEPs, separately for alpha, beta, and sham tACS sessions. Relevance values of these three fits were tested against permutationbased null distributions. These analyses revealed a significant effect for beta $\left(p=.011<\alpha_{\text {bonf }}\right)$, but not alpha $\left(p=.58>\alpha_{\text {bonf }}\right)$ or sham $\left(p=.11>\alpha_{\text {bonf }}\right)$ tACS, see Fig. 3 A. The post-hoc analysis for low and high beta groups revealed statistically significant fits in only the low beta group ( $p=.002<\alpha_{\text {bonf }}$ ), but not the high beta group ( $p=.57>\alpha_{\text {bonf, }}$, see Fig. $\left.3 \mathrm{~B}\right)$.

To rule out that these results were based only on a minor subgroup of participants, we performed a cross-validation analysis (see methods). Results showed no significant effects for alpha $\left(p=.53>\alpha_{\text {bonf }}\right)$, sham $\left(p=.42>\alpha_{\text {bonf }}\right)$, or beta tACS $\left(p=.05>\alpha_{\text {bonf }}\right)$. Again, the post-hoc analysis splitting up high and low beta groups revealed only an effect for the lower beta $\left(p<.013<\alpha_{\text {bonf }}\right)$, not the higher beta $\left(p=.96>\alpha_{\text {bonf }}\right)$, group.

Lastly, we performed an individual participant analysis, meaning that we extracted relevance values, and created null distributions of permuted relevance values, for each participant in each tACS condition. We compared the non-permuted relevance values with medians of those null distributions using the Wilcoxon signed-rank test. This analysis revealed

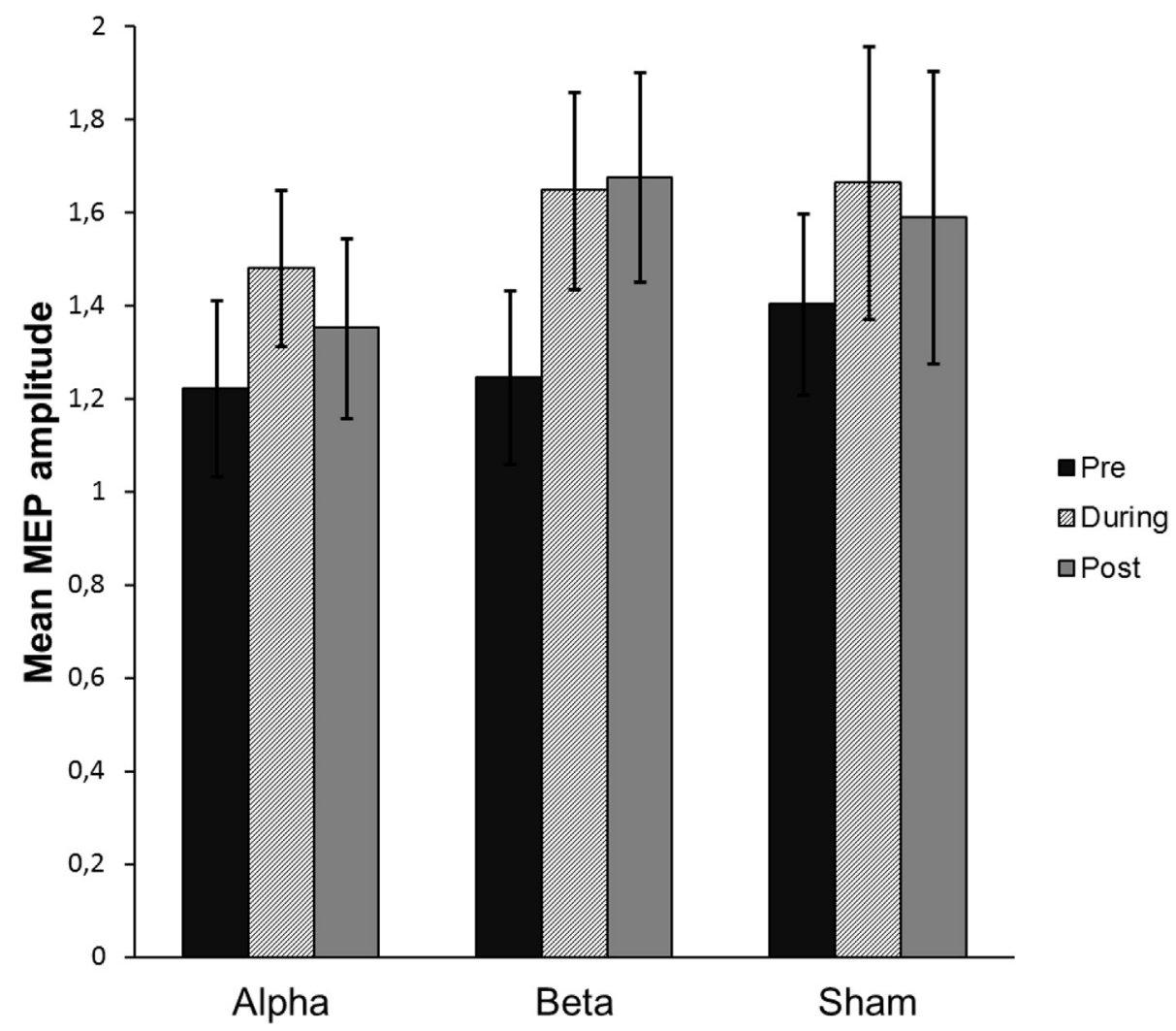

Fig. 2 - Results of the effect of either individual alpha, individual beta or sham entrainment on mean MEP amplitude. 
A

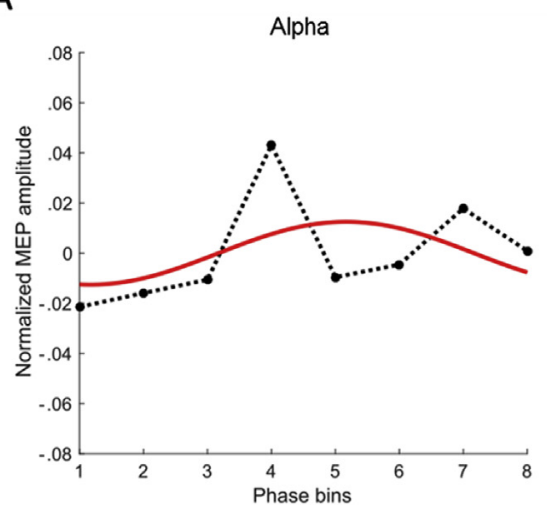

B

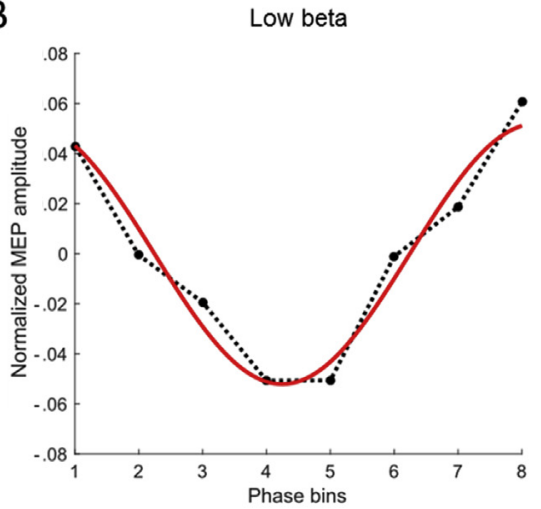

Beta

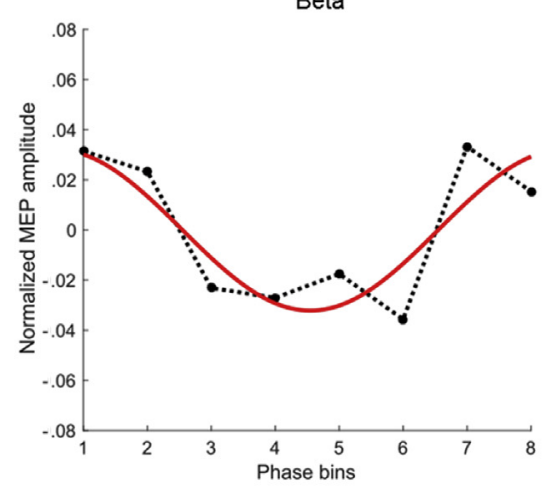

High beta

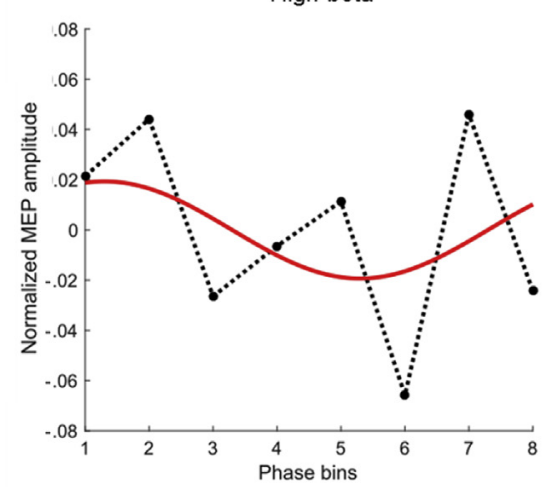

Sham

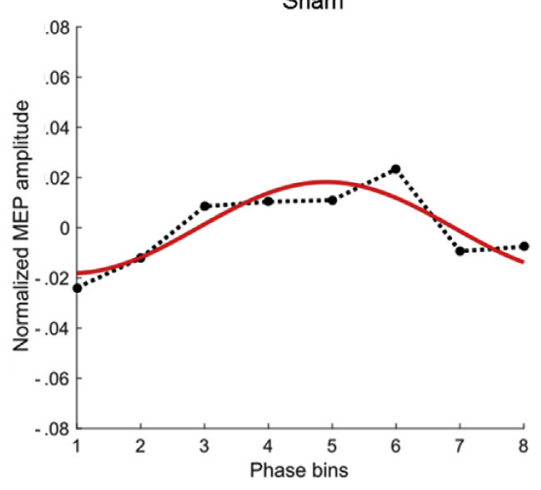

Fig. 3 - (A) Results of the group curve fitting analysis for alpha, beta, and sham and for (B) the high and low beta subgroups. The solid red lines represent the best fitting one-cycle sinusoid. The dotted black lines represent the mean MEP amplitude at different phases of the entraining tACS signal.

no significant effects for either alpha $\left(\mathrm{T}=84, p=.09>\alpha_{\mathrm{bonf}}\right)$, beta $\left(\mathrm{T}=55, p=.58>\alpha_{\text {bonf }}\right)$ or sham $\left(\mathrm{T}=65, p=.40>\alpha_{\text {bonf }}\right)$. For both high and low beta this result was also not significant ( $\mathrm{T}=10, p=.88>\alpha_{\mathrm{bonf}}$ and $\mathrm{T}=20, p=.19>\alpha_{\mathrm{bonf}}$ respectively).

\section{Discussion}

We combined single pulse TMS and EMG with tACS at individual alpha and beta frequencies to examine frequency- and phase-dependent effects of online tACS on corticospinal excitability. We found that the magnitude of MEP amplitude was affected by tACS in a phase-dependent manner for individual peak beta frequency tACS application at the time of the TMS pulse. Specifically, group averaged MEP amplitudes displayed a sinusoidal pattern over sequential phase conditions of online tACS. Moreover, post-hoc analyses suggested that this phase-dependence was specific to individuals with low individual beta frequencies $(16-19 \mathrm{~Hz}$ ), as opposed to high beta frequencies $(19-25 \mathrm{~Hz})$. These results indicate that corticospinal excitability quantified by MEP amplitudes is dependent on both phase and frequency of the ongoing tACS signal at the dominant intrinsic frequency within the beta band, conceivably the low beta band. We did not find frequency-specific offline effects of tACS.

\subsection{The effect of tACS at alpha and beta frequency on corticospinal excitability}

For the investigation of general offline and online effects of the simultaneous tACS-TMS paradigm we compared measures of corticospinal excitability acquired before, during and after alpha, beta, and sham tACS. When testing for changes in corticospinal excitability, only a main effect of time was observed, resulting from a general increase of MEP amplitude in all sessions. We did not observe a modulatory effect on MEPs during or after tACS-TMS stimulation. This finding is in line with other studies reporting no modulatory effects on MEPs during or after tACS over M1 at (individual) beta or other frequencies (Antal et al., 2008; Nowak et al., 2017; Wach et al., 2013). In contrast, yet other studies have reported such modulatory effects (Feurra et al., 2011, 2013; Schutter \& Hortensius, 2011), however their design differed to that of the current study. We applied tACS in combination with TMS at individual peak frequencies, for a much longer duration (36.5 $\mathrm{min}$ ) and with the stimulation intensity for tACS being $50 \%$ higher (1.5 mA). Moreover, we compared stimulation effects to sham effects and found no difference between both stimulation protocols. These differences in the application of tACS might explain the discrepancy between results. Additionally, Pellicciari and colleagues recently showed that 
administering single TMS pulses to motor cortex has cumulative effects that result in increased MEP amplitudes for both fixed and randomized intervals (Pellicciari, Miniussi, Ferrari, Koch, \& Bortoletto, 2016). Although the inter pulse interval in the current experiment was longer compared to the aforementioned study (6.75 sec compared to $44 \mathrm{sec}$ on average), it may still be possible that such cumulative TMS stimulation effects overruled any possible modulatory effect of tACS application on MEP amplitudes during and after tACS. In line with our experimental design and results, Raco and colleagues showed that combining beta-tACS with single pulse TMS had cumulative effects on MEP amplitude exclusively when applied at 120\% MT (Raco, Bauer, Norim, \& Gharabaghi, 2017). Therefore, we cannot rule out that tACS application alone could have frequency-specific modulatory effects on MEP amplitudes.

\subsection{The effect of tACS phase on corticospinal excitability}

Our results suggest that corticospinal excitability is modulated by ongoing tACS in a phase-dependent manner. Average MEP amplitudes elicited at eight equidistant tACS phases displayed a sinusoidal pattern when tACS was applied at individual beta frequencies, but not at individual alpha frequencies or when sham-tACS was applied. This shows that MEP amplitude is dependent on the phase of ongoing individually-calibrated beta-frequency tACS. Our findings are in line with previous studies that reported fixed frequency beta tACS $(20 \mathrm{~Hz})$ phase-dependent TMS-induced MEP amplitude modulations (Raco et al., 2016), but failed to find such effects at non-motor resonance frequencies (Guerra et al., 2016; Nakazono, Ogata, Kuroda, \& Tobimatsu, 2016). Moreover, in contrast to previous studies, our experimental design (wide range of measured phases) and analyses (sinusoidal curve fitting) could directly show, for the first time, an oscillatory pattern of acquired MEPs. This supports a direct and continuous influence of beta tACS phase on corticospinal excitability. Moreover, post-hoc analyses in which we divided the data based on intrinsic high $(>19 \mathrm{~Hz})$ or low $(<19 \mathrm{~Hz})$ beta frequency, showed that this tACS phase-dependence was highly significant in the low, but not the high beta group.

\subsection{The potential influence of intrinsic natural oscillations on cortical and corticospinal excitability}

Momentary state of spontaneous neuronal oscillations has been related repeatedly to cortical or corticospinal excitability. Several simultaneous TMS-EEG studies suggest that natural oscillatory power and phase affect corticospinal excitability (Keil et al., 2014; Lepage, Saint-Amour, \& Théoret, 2008; Mäki \& Ilmoniemi, 2010; Schulz, Übelacker, Keil, Müller \& Weisz., 2014). Schulz et al., (2014) reported a negative correlation between MEP amplitude and high pre-pulse beta-band power in the contralateral motor, premotor, parietal and frontal areas, as well as in the ipsilateral temporal areas. This finding is in accordance with other studies showing a reduction of MEP amplitudes with increasing beta band power, especially for low beta frequencies (12-18 Hz) (Lepage et al., 2008; Mäki \& Ilmoniemi, 2010). In addition, TMS-induced MEP amplitudes have shown to increase when TMS pulses are applied at either the peak or the trough of the beta oscillation compared to other phase bins (Keil et al., 2014). Negative versus positive peaks of sensorimotor intrinsic neuronal oscillations at alpha frequency ( $\mu$-rhythm) have been associated with high versus low corticospinal excitability states (Zrenner, Desideri, Belardinelli, \& Ziemann, 2017). Elevated MEP amplitude has also been associated with an increase of pre-stimulus corticomuscular coherence in alpha and beta frequencies at several motor activity relevant areas. In addition, the combination of low pre-stimulus beta band power along with high alpha band corticomuscular coherence has been related to high MEPs (and vice versa) (Schulz et al., 2014).

\subsection{Specificity of phase dependence in the low beta frequency range}

The association of corticospinal excitability with beta band tACS frequency is in line with the idea that brain oscillations in the beta frequency are related to the preparation and execution of movement (Neuper \& Pfurtscheller, 2001; Pfurtscheller, 1981). With regard to both limb movement and somatosensory stimulation, beta frequency has been reported to be lower over cortical representations of the hand area (below $20 \mathrm{~Hz}$ ) than of the foot area (above $20 \mathrm{~Hz}$ ) (Neuper \& Pfurtscheller, 2001). This could explain why we found phase dependency especially for lower beta band tACS application, given that MEPs were measured from the FDI muscle of the hand. On the other hand, it is also possible that tACS application at the individual peak frequency from within the low beta band would have resulted in phase-dependent effects in all participants, including those who had a high individual peak beta frequency when tested or the grand beta band. Thus, tACS at low beta frequency could also be beneficial irrespectively of the intrinsic peak beta frequency. Alternatively, it is possible that phase-dependent effects are only present for individuals with an intrinsic low peak beta. Based on our data these possibilities cannot be distinguished.

\subsection{Individual peak frequencies}

Natural peak frequencies seem to be a crucial factor for cortical and corticospinal excitability. Using rhythmic repetitive TMS (rTMS), Romei et al. (2016); Romei, Thut, et al. (2016) demonstrated that elicited responses are strongest when stimulation is applied at the intrinsic individual peak beta frequency. In addition, tACS at intrinsic peak frequencies of specific frequency bands appears to be an important factor that could play a major role in (beta) entrainment efficacy (Davis, Tomlinson, \& Morgan, 2012).

In the current study, we applied tACS at the intrinsic peak frequency. With respect to previous research on tACS entrainment effects, this could have resulted in the entrainment of the applied frequency in the underlying cortical areas (Antal \& Paulus, 2013; Fröhlich \& McCormick, 2010; Herrmann et al., 2013; Witkowski et al., 2016). Indeed, entrainment at a frequency that is close to the underlying intrinsic frequency leads to the strongest increase in frequency power (Fröhlich \& McCormick, 2010; Merlet et al., 2013). Thus, by applying tACS at the intrinsic peak frequency it seems reasonable that tACS phase-specific TMS is also associated with the natural peak 
frequency. tACS-induced modulation of corticospinal excitability could therefore be linked to the frequency and phase of intrinsic neuronal oscillations. Therefore, measuring individual peak beta frequency and linking TMS to both this frequency and a particular phase of the tACS signal could be most beneficial for robust and meaningful measures of corticospinal excitability.

\subsection{Limitations}

In line with our hypothesis, the results of the current experiment showed a striking correspondence of the beta group MEP pattern over eight tACS phase bins to a one-cycle sinusoid. Nonetheless, it is important to also be aware of the limitations of our results. Based on previous research it seems reasonable to hypothesize that tACS leads to an entrainment of the applied frequency oscillation in underlying neuronal networks (Antal \& Paulus, 2013; Fröhlich \& McCormick, 2010; Herrmann et al., 2013; Neuling et al., 2013; Witkowski et al., 2016; Zaehle et al., 2010). By applying TMS phase-locked to the tACS signal over the primary motor cortex, we could assume that TMS is also phase locked to the entrained underlying neuronal oscillation. Thus, if this were the case, our results would suggest that corticospinal excitability is dependent on the current state of the underlying neuronal oscillation at the time and location of stimulation and that the driving agents in modulating excitability in the motor system are neuronal oscillations in the beta frequency range. However, we did not control for a tACS entrainment effects by simultaneously recording ongoing neuronal oscillation signals. Therefore, we can only draw conclusions about the effects of specific tACS frequencies and phases on TMS-induced MEP measures of corticospinal excitability. Future empirical studies, specifically also assessing tACS entrainment efficacy with EEG, need to be conducted in order to draw conclusions about the causal relevance of the frequency and phase of underlying neuronal oscillations for corticospinal excitability.

We report a general increase of MEP amplitude over time across all three visits (alpha, beta and sham) and conclude that we do not find modulatory effects of tACS at either stimulation frequency. However, we cannot rule out that periodical spTMS (although at jittered inter pulse intervals around $7 \mathrm{sec}$ ) enhances corticomuscular coherence, which has previously been demonstrated to have an effect on MEP amplitudes (Schulz et al., 2014). Therefore, the general increase of MEP amplitude may be caused by periodic spTMS-triggered enhanced corticomuscular coherence, thereby overruling any possible modulatory effect of tACS. Thus, a frequency-specific modulatory effect of tACS on MEP amplitude may still exist when tACS is applied without simultaneous TMS.

Curve-fitting in the beta group only showed a trend in the cross validation. Also, neither of the beta groups (all participants or low-beta participants) yielded significant results in the individual participant analysis that fit sinusoids to individual participant data. This asks for caution both when generalizing the results to individual corticospinal excitability assessments and when thinking of applying them to increase reliability of TMS measures of corticospinal excitability.

We determined individual dominant alpha frequency by analyzing resting EEG measures while participants kept their eyes closed. However, tACS at individual alpha peak frequencies was applied when participants kept their eyes open. It is therefore possible that the individual alpha peak frequencies used for tACS do not exactly correspond to the intrinsic peak alpha frequency when eyes are kept open. Moreover, alpha oscillations measured from resting EEG with eyes closed are prominent over posterior cortical areas. Thus, it is possible that the recorded peak alpha frequencies from the central electrode (C3) correspond to neuronal activity from posterior cortical areas instead of neuronal activity from the motor cortex. This might explain why no significant effects were observed in the alpha condition.

\subsection{Methodological implication}

In the present study we demonstrate that TMS measures of corticospinal excitability are influenced by time coupling of the TMS pulse with tACS frequency and phase. Previous studies showed that tACS successfully entrains oscillations of underlying neuronal networks. Together, these findings indicate that corticospinal excitability may depend on the phase of the intrinsic peak beta frequency, predominantly on the lower beta frequency band (16-19 Hz). Most studies investigating corticospinal excitability by measuring TMS-induced MEPs do not control for the state of the brain during stimulation. With respect to previous research and under restriction of the discussed limitations, our findings indicate that study outcomes may be influenced by fluctuations of corticospinal excitability, if the state of the brain is not controlled for, or at least not accounted for, when corticospinal excitability is measured. In addition, it stands to reason that local oscillation phase and frequency at the time of TMS administration may not only be crucial for the investigation of corticospinal excitability, but also for other forms of TMS application, such as motor plasticity inducing stimulation protocols or research on brain regions other than M1. Further investigations of frequency and phase-dependence of TMS efficacy could possibly help to explain recently accented high variability of TMS effects both across and within individuals (Schilberg, Schuhmann, \& Sack, 2017) and lead to more robustness of TMS measures. In order to acquire meaningful and reliable results it is important to carefully investigate underlying brain mechanisms that could influence TMS effects and to improve existing or to develop new application techniques. Incorporating measures of functional, physiological and anatomical properties of targeted neuronal networks into TMS protocols introduces a new era of information based approaches of non-invasive transcranial brain stimulation, which aim for enhanced specificity of stimulation (Romei et al, 2016; Romei, Thut, et al, 2016). This is not only crucial for fundamental research, but also for clinical applications of TMS in which high efficacy of applied protocols is necessary for a successful therapy of a disease or the reliable monitoring of its course.

\section{Author contribution}

Design of the experiment: LS TE TS TdG AS. Data collection: LS TE. Analysis and interpretation of the data: LS TE StO TdG. Writing of the manuscript: LS TE StO TS BdG TdG AS. 


\section{Acknowledgements}

This work was supported by the Netherlands Organization for Scientific Research (NWO: 453-15-008 and VENI: 451-13-024) and the European Research Council under the European Union's Seventh Framework Programme (FP7/2007-2013/ERC Grant agreement number 295673).

\section{R EFER E N C E S}

Antal, A., Boros, K., Poreisz, C., Chaieb, L., Terney, D., \& Paulus, W. (2008). Comparatively weak after-effects of transcranial alternating current stimulation (tACS) on cortical excitability in humans. Brain Stimulation, 1(2), 97-105. https://doi.org/ 10.1016/j.brs.2007.10.001.

Antal, A., \& Paulus, W. (2013). Transcranial alternating current stimulation (tACS). Frontiers in Human Neuroscience, 7, 317. https://doi.org/10.3389/fnhum.2013.00317.

Bestmann, S., \& Krakauer, J. W. (2014). The uses and interpretations of the motor-evoked potential for understanding behaviour. Experimental Brain Research, 233(3), 679-689. https://doi.org/10.1007/s00221-014-4183-7.

Buzsáki, G., \& Draguhn, A. (2004). Neural oscillations in cortical networks. Science, 304(5679), 1926-1929. https://doi.org/ 10.1126/science.1099745.

Chen, R., Kumar, S., Garg, R. R., \& Lang, A. E. (2001). Impairment of motor cortex activation and deactivation in Parkinson's disease. Clinical Neurophysiology, 112(4), 600-607. https:// doi.org/10.1016/S1388-2457(01)00466-7.

Davis, N. J., Tomlinson, S. P., \& Morgan, H. M. (2012). The role of beta-frequency neural oscillations in motor control. Journal of Neuroscience, 32(2), 403-404. https://doi.org/10.1523/ JNEUROSCI.5106-11.2012.

Di Lazzaro, V., Oliviero, A., Pilato, F., Saturno, E., Dileone, E., Marra, C., et al. (2004). Motor cortex hyperexcitability to transcranial magnetic stimulation in Alzheimer's disease. Journal of Neurology Neurosurgery and Psychiatry, 75, 555-559. https://doi.org/10.1136/jnnp.2003.018127.

Di Lazzaro, V., Oliviero, A., Profice, P., Ferrara, L., Saturno, E., Pilato, F., et al. (1999). The diagnostic value of motor evoked potentials. Clinical Neurophysiology, 110(7), 1297-1307. https:// doi.org/10.1016/S1388-2457(99)00060-7.

Ferreri, F., Pauri, F., Pasqualetti, P., Fini, R., Dal Forno, G., \& Rossini, P. M. (2002). Motor cortex excitability in Alzheimer's disease: A transcranial magnetic stimulation study. Annals of Neurology, 53(1), 102-108. https://doi.org/10.1002/ana.10416.

Feurra, M., Bianco, G., Santarnecchi, E., Del Testa, M., Rossi, A., \& Rossi, S. (2011). Frequency-dependent tuning of the human motor system induced by transcranial oscillatory potentials. Journal of Neuroscience, 31(34), 12165-12170. https://doi.org/ 10.1523/JNEUROSCI.0978-11.2011.

Feurra, M., Pasqualetti, P., Bianco, G., Santarnecchi, E., Rossi, A., \& Rossi, S. (2013). State-dependent effects of transcranial oscillatory currents on the motor System: What you think matters. Journal of Neuroscience, 33(44), 17483-17489. https:// doi.org/10.1523/JNEUROSCI.1414-13.2013.

Fiebelkorn, I. C., Foxe, J. J., Butler, J. S., Mercier, M. R., Snyder, A. C., \& Molholm, S. (2011). Ready, set, reset: Stimuluslocked periodicity in behavioral performance demonstrates the consequences of cross-sensory phase reset. Journal of Neuroscience, 31(27), 9971-9981. https://doi.org/10.1523/ JNEUROSCI.1338-11.2011.

Fröhlich, F., \& McCormick, D. A. (2010). Endogenous electric fields may guide neocortical network activity. Neuron, 67(1), 129-143. https://doi.org/10.1016/j.neuron.2010.06.005.
Fuhr, P., Borggrefe-Chappuis, A., Schindler, C., \& Kappos, L. (2001). Visual and motor evoked potentials in the course of multiple sclerosis. Brain, 124(11), 2162-2168. https://doi.org/10.1093/ brain/124.11.2162.

Guerra, A., Pogosyan, A., Nowak, M., Tan, H., Ferreri, F., Di Lazzaro, V., et al. (2016). Phase dependency of the human primary motor cortex and cholinergic inhibition cancelation during beta tACS. Cerebral Cortex, 26(10), 3977-3990. https:// doi.org/10.1093/cercor/bhw245.

Herrmann, C. S., Rach, S., Neuling, T., \& Strüber, D. (2013). Transcranial alternating current stimulation: A review of the underlying mechanisms and modulation of cognitive processes. Frontiers in Human Neuroscience, 7(279). https:// doi.org/10.3389/fnhum.2013.00279.

Jensen, O., \& Mazaheri, A. (2010). Shaping functional architecture by oscillatory alpha activity: Gating by inhibition. Frontiers in Human Neuroscience, 4(186). https://doi.org/10.3389/ fnhum.2010.00186.

Jung, N. H., Delvendahl, I., Kuhnke, N. G., Hauschke, D., Stolle, S., \& Mall, V. (2010). Navigated transcranial magnetic stimulation does not decrease the variability of motor-evoked potentials. Brain Stimulation, 3(2), 87-94. https://doi.org/10.1016/ j.brs.2009.10.003.

Jurkiewicz, M. T., Gaetz, W. C., Bostan, A. C., \& Cheyne, D. (2006). Post-movement beta rebound is generated in motor cortex: Evidence from neuromagnetic recordings. Neuroimage, 32(3), 1281-1289. https://doi.org/10.1016/j.neuroimage.2006.06.005.

Kallmann, B. A., Fackelmann, S., Toyka, K. V., Riekmann, P., \& Reiners, K. (2006). Early abnormalities of evoked potentials and future disability in patients with multiple sclerosis. Multiple Sclerosis Journal, 12(1), 58-65. https://doi.org/10.1191/ 135248506 ms1244oa.

Keil, J., Timm, J., Sanmiguel, I., Schulz, H., Obleser, J., \& Schönwiesner, M. (2014). Cortical brain states and corticospinal synchronization influence TMS-evoked motor potentials. Journal of Neurophysiology, 111(3), 513-519. https:// doi.org/10.1152/jn.00387.2013.

Kiers, L., Cros, D., Chiappa, K. H., \& Fang, J. (1993). Variability of motor potentials evoked by transcranial magnetic stimulation. Electroencephalography and Clinical Neurophysiology Evoked Potentials, 89(6), 415-423. https://doi.org/10.1016/01685597(93)90115-6.

Kilavik, B., Zaepffel, M., Brovelli, A., Mackay, W., \& Riehle, A. (2013). The ups and downs of beta oscillations in sensorimotor cortex. Experimental Neurology, 245, 15-26. https://doi.org/ 10.10167/j.expneurol.2012.09.014.

Lefaucheur, J. (2005). Motor cortex dysfunction revealed by cortical excitability studies in Parkinson's disease: Influence of antiparkinsonian treatment and cortical stimulation. Clinical Neurophysiology, 116(2), 244-253. https://doi.org/10.1016/ j.clinph.2004.11.017.

Lepage, J. F., Saint-Amour, D., \& Théoret, H. (2008). EEG and neuronavigated single-pulse TMS in the study of the observation/execution matching system: Are both techniques measuring the same process? Journal of Neuroscience Methods, 175(1), 17-24. https://doi.org/10.1016/j.jneumeth.2008.07.021.

Mäki, H., \& Ilmoniemi, R. J. (2010). EEG oscillations and magnetically evoked motor potentials reflect motor system excitability in overlapping neuronal populations. Clinical Neurophysiology, 121(4), 492-550. https://doi.org/10.1016/ j.clinph.2009.11.078.

Mathewson, K. E., Lleras, A., Beck, D. M., Fabiani, M., Ro, T., \& Gratton, G. (2011). Pulsed out of awareness: EEG alpha oscillations represent a pulsed-inhibition of ongoing cortical processing. Frontiers in Psychology, 2(99), 1-15. https://doi.org/ 10.3389/fpsyg.2011.00099.

Merlet, I., Birot, G., Salvador, R., Molaee-Ardekani, B., Mekonnen, A., Soria-Frish, A., et al. (2013). From oscillatory 
transcranial current stimulation to scalp EEG changes: A biophysical and physiological modeling study. PloS One, 8(2). https://doi.org/10.1371/journal.pone.0057330.

Moliadze, V., Fritzsche, G., \& Antal, A. (2014). Comparing the efficacy of excitatory transcranial stimulation methods measuring motor evoked potentials. Neural Plasticity, 2014(837141). https://doi.org/10.1155/2014/837141.

Nakazono, H., Ogata, K., Kuroda, T., \& Tobimatsu, S. (2016). Phase and frequency-dependent effects of transcranial alternating current stimulation on motor cortical excitability. PloS One, 11(9). https://doi.org/10.1371/journal.pone.0162521. e0162521.

Neuling, T., Rach, S., \& Herrmann, C. S. (2013). Orchestrating neuronal networks: Sustained after-effects of transcranial alternating current stimulation depend upon brain states. Frontiers in Human Neuroscience, 7, 161. https://doi.org/10.3389/ fnhum.2013.00161.

Neuper, C., \& Pfurtscheller, G. (2001). Evidence for distinct beta resonance frequencies in human EEG related to specific sensorimotor cortical areas. Clinical Neurophysiology, 112(11), 2084-2097. https://doi.org/10.1016/S1388-2457(01)00661-7.

Ni, Z., \& Chen, R. (2015). Transcranial magnetic stimulation to understand pathophysiology and as potential treatment for neurodegenerative diseases. Translational Neurodegeneration, 4, 22. https://doi.org/10.1186/s40035-015-0045-x.

Niedermeyer, E. (2005). The normal EEG of the waking adult. Electroencephalography: Basic principles, clinical applications and related field (Vol. 167, pp. 155-164). Baltimore: Lippincott, Williams \& Wilkins.

Nowak, M., Hinson, E., van Ede, F., Pogosvan, A., Guerra, A., Quinn, A., et al. (2017). Driving human motor cortical oscillations leads to behaviorally relevant changes in local GABA(a) inhibition: A tACS-TMS study. The Journal of Neuroscience, 37(17), 4481-4492. https://doi.org/10.1523/ JNEUROSCI.0098-17.2017.

Oostenveld, R., Fries, P., Maris, E., \& Schoffelen, J. M. (2011). FieldTrip: Open source software for advanced analysis of MEG, EEG, and invasive electrophysiological data. Computational Intelligence and Neuroscience, 2011(156869), 1-9. https://doi.org/ 10.1155/2011/156869.

Pascual-Leone, A., Tormos, J., \& Keenan, J. (1998). Study and modulation of human cortical excitability with transcranial magnetic stimulation. Journal of Clinical Neurophysiology, 15(4), 333-343.

Pellicciari, M. C., Miniussi, C., Ferrari, C., Koch, G., \& Bortoletto, M. (2016). Ongoing cumulative effects of single TMS pulses on corticospinal excitability: An intra- and inter-block investigation. Clinical Neurophysiology, 127(1), 621-628. https:// doi.org/10.1016/j.clinph.2015.03.002.

Pfurtscheller, G. (1981). Central beta rhythm during sensorimotor activities in man. Electroencephalography and Clinical Neurophysiology, 51(3), 253-264. https://doi.org/10.1016/00134694(81)90139-5.

Pfurtscheller, G., Stancák, A., \& Neuper, C. (1996). Post-movement beta synchronization. A correlate of an idling motor area? Electroencephalography and Clinical Neurophysiology, 98(4), 281-293. https://doi.org/10.1016/0013-4694(95)00258-8.

Raco, V., Bauer, R., Norim, S., \& Gharabaghi, A. (2017). Cumulative effects of single TMS pulses during beta-tACS are stimulation intensity-dependent. Brain Stimulation, 10(6), 1055-1060. https://doi.org/10.16/j.brs.2017.07.009.

Raco, V., Bauer, R., Tharsan, S., \& Gharabaghi, A. (2016). Combining TMS and tACS for closed-loop phase-dependent modulation of corticospinal excitability: A feasibility study. Frontiers in Cellular Neuroscience, 10(143), 1-8. https://doi.org/ 10.3389/fncel.2016.00143.

Rico, A., Audoin, B., Frangues, J., Eusebio, A., Reuter, F., Malikova, I., et al. (2009). Motor evoked potentials in clinically isolated syndrome suggestive of multiple sclerosis. Multiple
Sclerosis Clinical and Laboratory Research, 15(3), 355-362. https:// doi.org/10.1177/1352458508099612.

Romei, V., Bauer, M., Brooks, J. L., Economides, M., Penny, W., Thut, G., et al. (2016). Causal evidence that intrinsic betafrequency is relevant for enhanced signal propagation in the motor system as shown through rhythmic TMS. Neuroimage, 126, 120-130. https://doi.org/10.1016/ j.neuroimage.2015.11.020.

Romei, V., Thut, G., \& Silvanto, J. (2016). Information-based approaches of noninvasive transcranial brain stimulation. Trends in Neuroscience, 39(11), 782-795. https://doi.org/10.1016/ j.tins.2016.09.001.

Rösler, K. M., Roth, D. M., \& Magistris, M. R. (2008). Trial-to-trial size variability of motor-evoked potentials. A study using the triple stimulation technique. Experimental Brain Research, 187(1), 51-59. https://doi.org/10.1007/s00221-008-1278-z.

Rossi, S., Hallett, M., Rossini, P. M., \& Pascual-Leone, A. (2012). Safety, ethical considerations, and application guidelines for the use of transcranial magnetic stimulation in clinical practice and research. Clinical Neurophysiology, 120(12), 323-330. https://doi.org/10.1016/j.clinph.2009.08.016.Rossi.

Rossini, P. M., \& Rossi, S. (1998). Clinical applications of motor evoked potentials. Clinical Neurophysiology, 106(3), 180-194. https://doi.org/10.1016/S0013-4694(97)00097-7.

Rothwell, J. C., Hallett, M., Berardelli, A., Eisen, A., Rossini, P., \& Paulus, W. (1999). Magnetic stimulation: Motor evoked potentials. The International federation of clinical neurophysiology. Electroencephalography and Clinical Neurophysiology Supplement, 52, 97-103.

Schilberg, L., Schuhmann, T., \& Sack, A. T. (2017). Interindividual variability and intraindividual reliability of intermittent theta burst stimulation-induced neuroplasticity mechanisms in the healthy brain. Journal of Cognitive Neuroscience, 29(6), 1022-1032. https://doi.org/10.1162/jocn_a_01100.

Schulz, H., Übelacker, T., Keil, J., Müller, N., \& Weisz, N. (2014). Now I am ready - now i am not: The influence of pre-TMS oscillations and corticomuscular coherence on motor-evoked potentials. Cerebral Cortex, 24(7), 1708-1719. https://doi.org/ 10.1093/cercor/bht024.

Schutter, D. J. L. G., \& Hortensius, R. (2011). Brain oscillations and frequency-dependent modulation of cortical excitability. Brain Stimulation, 4(2), 97-103. https://doi.org/10.1016/ j.brs.2010.07.002.

Ten Oever, S., de Graaf, T. A., Bonnemayer, C., Ronner, J., Sack, A. T., \& Riecke, L. (2016). Stimulus presentation at specific neuronal oscillatory phases experimentally controlled with tACS: Implementation and applications. Frontiers in Cellular Neuroscience, 10(240), 1-8. https://doi.org/10.3389/ fncel.2016.00240.

Wach, C., Krause, V., Moliadze, V., Paulus, W., Schnitzler, A., \& Pollok, B. (2013). Effects of $10 \mathrm{~Hz}$ and $20 \mathrm{~Hz}$ transcranial alternating current stimulation (tACS) on motor functions and motor cortical excitability. Behavioural Brain Research, 241, 1-6. https://doi.org/10.1016/j.bbr.2012.11.038.

Witkowski, M., Garcia-Cossio, E., Chander, B. S., Braun, C., Birbaumer, N., Robinson, S. E., et al. (2016). Mapping entrained brain oscillations during transcranial alternating current stimulation (tACS). Neuroimage, 140, 89-98. https://doi.org/ 10.1016/j.neuroimage.2015.10.024.

Zaehle, T., Rach, S., \& Herrmann, C. S. (2010). Transcranial alternating current stimulation enhances individual alpha activity in human EEG. PloS One, 5(11). https://doi.org/10.1371/ journal.pone.0013766.

Zrenner, C., Desideri, D., Belardinelli, P., \& Ziemann, U. (2017). Real-time EEG-defined excitability states determine efficacy of TMS-induced plasticity in human motor cortex. Brain Stimulation. https://doi.org/10.1016/j.brs.2017.11.016. 